\title{
Wnt signaling in development and disease
}

Yingzi Yang*

\begin{abstract}
Cell signaling mediated by morphogens is essential to coordinate growth and patterning, two key processes that govern the formation of a complex multi-cellular organism. During growth and patterning, cells are specified by both quantitative and directional information. While quantitative information regulates cell proliferation and differentiation, directional information is conveyed in the form of cell polarities instructed by local and global cues. Major morphogens like Wnts play critical roles in embryonic development and they are also important in maintaining tissue homeostasis. Abnormal regulation of these signaling events leads to a diverse array of devastating diseases including cancer. Wnts transduce their signals through several distinct pathways and they regulate vertebrate embryonic development by providing both quantitative and directional information. Here, taking the developing skeletal system as an example, we review our work on Wnt signaling pathways in various aspects of development. We focus particularly on our most recent findings that showed that in vertebrates, Wnt5a acts as a global cue to establishing planar cell polarity (PCP). Our work suggests that Wnt morphogens regulate development by integrating quantitative and directional information. Our work also provides important insights in disease like Robinow syndrome, brachydactyly type B1 (BDB1) and spina bifida, which can be caused by human mutations in the Wnt/PCP signaling pathway.
\end{abstract}

\section{Brief introduction of Wnt signaling pathways in development and disease}

One of the most remarkable biological processes is the formation of a morphologically complex and functional diverse multicellular organism such as a human being from a single fertilized egg within a short period of time. During this tightly regulated process of embryonic morphogenesis, functional tissues and organs are formed and they have to be properly maintained during adult lives. Because cell-cell signaling plays essential and pivotal roles in both embryonic development and adult physiology, understanding the function and the underlying molecular mechanism of key cell signaling pathways in both development and diseases has been a major focus of our lab. Here we primarily focus on Wnt signaling in major developmental events of the skeletal system.

Wnts are evolutionarily conserved major regulatory factors in both development and disease. Wht signaling is required in most embryonic developmental processes in both invertebrates and vertebrates. Abnormal Wnt signaling causes many types of tumors [1-3]. For instance, ectopic activation of Wnt1 gene expression in the mouse mammary

Correspondence: yingzi@mail.nih.gov

Genetic Disease Research Branch, National Human Genome Research Institute, 49 Convent DriveMSC 4472, Bethesda, MD 20892, USA gland leads to tumor formation [4]. Mutations in Wnt signaling components have also been found to cause other human diseases. Weakened Wnt/ $\beta$-catenin signaling leads to osteoporosis-pseudoglioma syndrome due to reduced bone mass [5] whereas enhanced $\mathrm{Wnt} / \beta$-catenin signaling causes thick bone syndrome due to increased bone mass [6,7]. In addition, Robinow syndrome and Brachydactyly Type B1 that are characterized by shortened skeletal elements are caused by mutations in the Wnt/planar cell polarity pathway components [8-11].

Wnts are a large family of secreted molecules that can signal through several distinct pathways (Figure 1). The $\beta$-catenin mediated $\mathrm{Wnt} / \beta$-catenin pathway is also called the canonical pathway. This pathway is best understood and mainly controls cell proliferation and differentiation. Central to this pathway is the control of $\beta$-catenin stability. In the absence of Wnt signaling, $\beta$-catenin is phosphorylated by GSK-3 in the "destruction complex" brought together by Axin and APC. Phosphorylated $\beta$ catenin was then recognized by the ubquitination machinery and sent to degradation in the proteosome. When Wnts bind to their receptors Frizzleds and Lrp5 or Lrp6, Lrp5/6 are phosphorylated and Dishevelled is activated, which lead to inactivation of the $\beta$-catenin "destruction complex" or disassembly of the $\beta$-catenin "destruction 


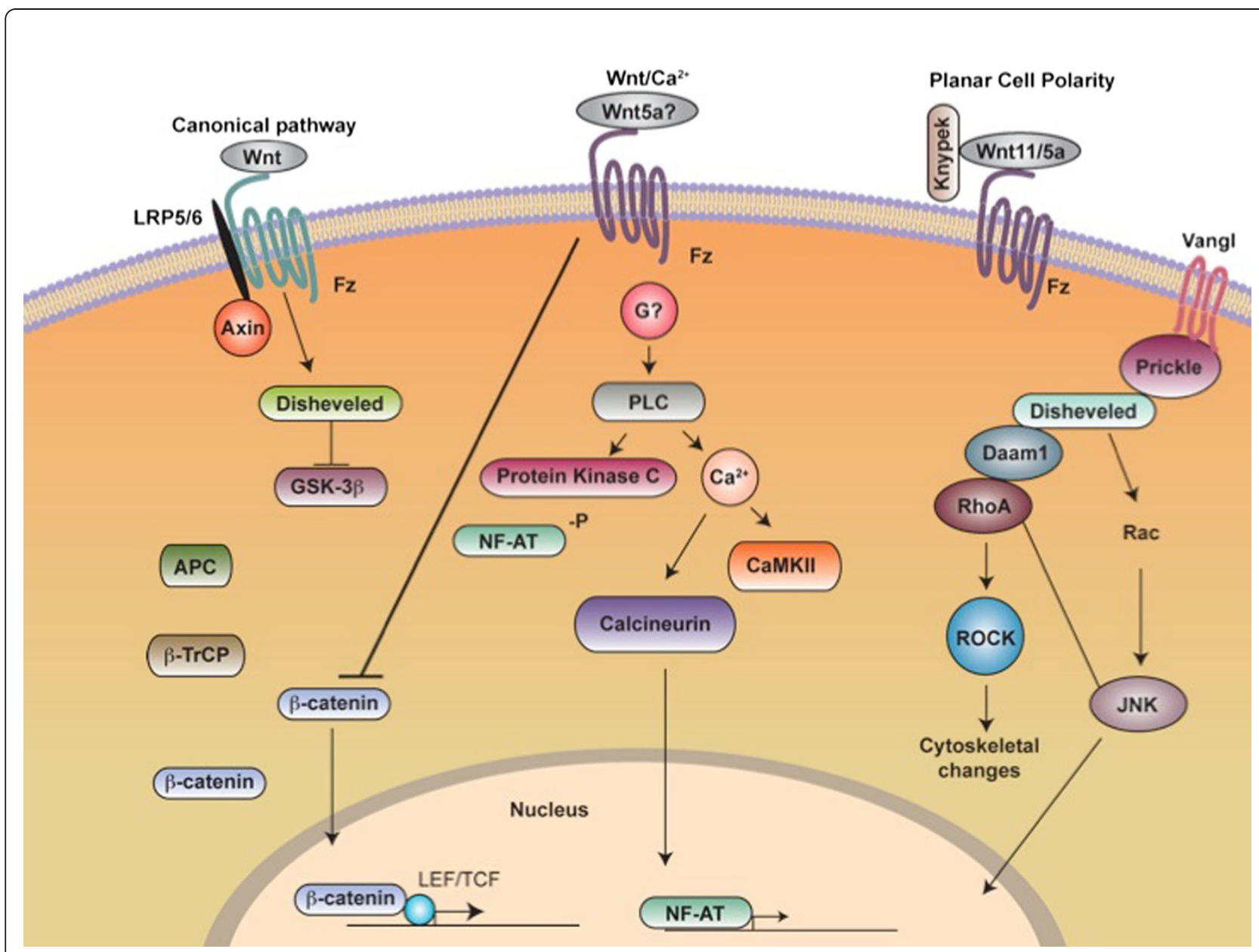

Figure 1 Three representative Wnt signaling pathways. See text for details.

complex" such that $\beta$-catenin phosphorylation is reduced and stabilized. The stabilized $\beta$-catenin then translocates to the nucleus where it regulates downstream gene expression by binding to Lef/Tcf factors. Wnts may also signal through regulating intracellular $\mathrm{Ca}++$ mobilization [12], but the regulation and functional significance of this pathway in mammalian development remain unclear. The planar cell polarity (PCP) pathway is also evolutionarily conserved and shares several components with the canonical pathway. Although Wnt ligands have not been found to regulate PCP in Drosophila [13], PCP is genetically regulated by Wnt5a and/or Wnt11 in vertebrates [14-16]. Recent findings demonstrate that Wnt signaling acts through this pathway to provide directional information in controlling morphogenesis [17-20]. This part will be elaborated below.

\section{The skeleton provides a great system to study the functional mechanisms of Wnt signaling in vivo}

The developing skeleton provides a good system to study major events in embryonic morphogenesis. It is relatively simple in its structure and cellular composition. It only contains three major cell types: chondrocytes that form the cartilage, osteoblasts that secrete bone matrix and osteoclasts that are specialized macrophage cells differentiated from hematopoietic precursor cells [21,22]. The skeletal system is widely distributed throughout the body, covering, supporting and protecting important organs. Compelling evidence also indicates that the skeletal system plays systemic regulatory roles as well [23]. The skeletal elements exhibit characteristic morphologies and organization that serve as convenient readouts for regulation of morphogenesis. For instance, the entire skeleton can be visualized in great detail in the intact embryo or adult animal after simple staining procedures.

Skeletal development starts from mesenchymal condensation in which mesenchymal progenitor cells are at least bipotential and they are also called osteochondral progenitors. These cells differentiate into either osteoblasts or chondrocytes depending on the mechanism of ossification. During intramembranous ossification that occurs primarily 
in the skull, osteochondral progenitors differentiate directly into bone forming osteoblasts whereas in endochondral ossification that occurs in most parts of the body, osteochondral progenitors first differentiate into cartilage forming chondrocytes instead. Osteoblast cells then form in the peripheral of the cartilage template and invade the hypertrophic cartilaginous area together with blood vessels to start the formation of trabecular bone. After bone formation, osteoclasts brought in by blood vessels remodels the bone, which is very important in maintaining bone homeostasis in adult lives [21]. The skeletal system is also highly segmented. Individual skeletal elements connect to each other through joints and this feature is required for motilities of vertebrate animals.

\section{The canonical Wnt signaling pathway controls cell fate determination in several fundamentally important processes of skeletal development}

When my lab first got into the field of skeletal biology a decade ago, I decided to start by investigating the regulatory mechanism underlying several fundamentally important processes of skeletal development: the determination of chondrocytes versus osteoblasts when osteochondral progenitor cells are differentiating; The sequential proliferation and hypertrophy of chondrocytes in the long bone cartilage and induction of synovial joint formation. Now we found that it is quite remarkable that all these fundamental processes are controlled by $\mathrm{Wnt} / \beta$-catenin signaling $[17,24-30]$. The functional spectrum of Wnt signaling in skeletal biology is getting broader and Wnt signaling has become a major field in skeletal biology.

To understand the functions of the canonical Wnt signaling in skeletal development, we utilized a canonical Wnt signaling reporter mouse strain Top-Gal in which lacZ expression reflects active canonical Wnt signaling. We found that in the Top-Gal mouse embryo, X-gal staining is selectively upregulated in the developing joint [28] marked by the expression of the earliest known joint marker Gdf5 [31]. LacZ expression is also detected in the calvarium and perichchondrium where osteoblasts differentiate through intramembranous or endochondral ossification, respectively [30]. These results suggest canonical Wnt signaling may be important in joint formation and/or osteoblast differentiation regardless of ossification mechanisms [28,30]. Indeed we found by genetic manipulation of canonical Wnt signaling that this signaling pathway is both necessary and sufficient to induce joint formation [28] (Figure 2). The canonical Wnt pathway also controls bone and cartilage formation by controlling cell fate determination regardless of the ossification mechanism [30](Figure 2).

Taken together, we have found that the mesenchymal progenitor cells in the condensation are bipotential $[28,30]$ (Figure 2). During intramembranous ossification, higher Wnt signaling in the condensation leads to inhibition of chondondrocyte differentiation and promotion of osteoblast differentiation. During endochondral ossification, however, Wnt signaling is kept low in the condensation such that only chondrocytes can differentiate. Later, when Wnt signaling is upregulated in the peripheral of the cartilage, osteoblasts will differentiate. Wnt signaling upregulation in the presumptive joint area induces joint formation. Therefore, by manipulating Wnt signaling, mesenchymal progenitor cells can be directed to form only chondrocytes or osteoblasts. These studies, together with others, have indicated that higher canonical Wnt signaling in cartilage may cause cartilage damages such as those observed in osteoarthritis [32]. Moreover, antibody therapies targeting canonical Wnt signaling are quite effective in increasing bone mass and are under clinical trials [33-36].

\section{The Wnt/PCP pathway controls skeletal morphogenesis by providing directional information}

One of the most important functions of secreted molecules, Wnts included, is to act as morphogens which are critically required in embryonic morphogenesis by coordinating cell proliferation with cell fate determination [37-39]. This function has been the focal attention of developmental biology. However, the entire organism as well as the internal tissues and organs exhibit distinct morphologies and organizations which are essential for their functions. To understand how these are generated during morphogenesis, one has to understand how directional or polarity information is provided globally and locally. If cells in the developing embryo were not polarized and could not sense global or local directional cues, proliferation and differentiation would be the predominant mechanisms driving the development of the embryo. The result would be a three-dimensionally symmetric sphere composed of many different cell types. Therefore, providing directional information is fundamentally important in biology. For example, it is important to understand why the limb preferentially elongates along the proximal-distal axis.

It is well understood that morphogens such as Wnts can form a concentration gradient across a field of hundreds of cells (Figure 3). This leads to the generation of different cell types in a distinct spatial order according to local threshold concentrations of the morphogen. In this regard, morphogen gradients provide quantitative information to generate a distinct pattern (Figure 3). For instance, the limb bud is patterned along the anterior-posterior and proximal-distal axes by morphogen gradients [40,41]. The fact that our limbs are elongated instead of being a three dimensionally symmetrical ball tells us that directional information has to be provided during pattern formation. However, it is unknown whether and how morphogen gradients also provide directional information (Figure 3). It is 

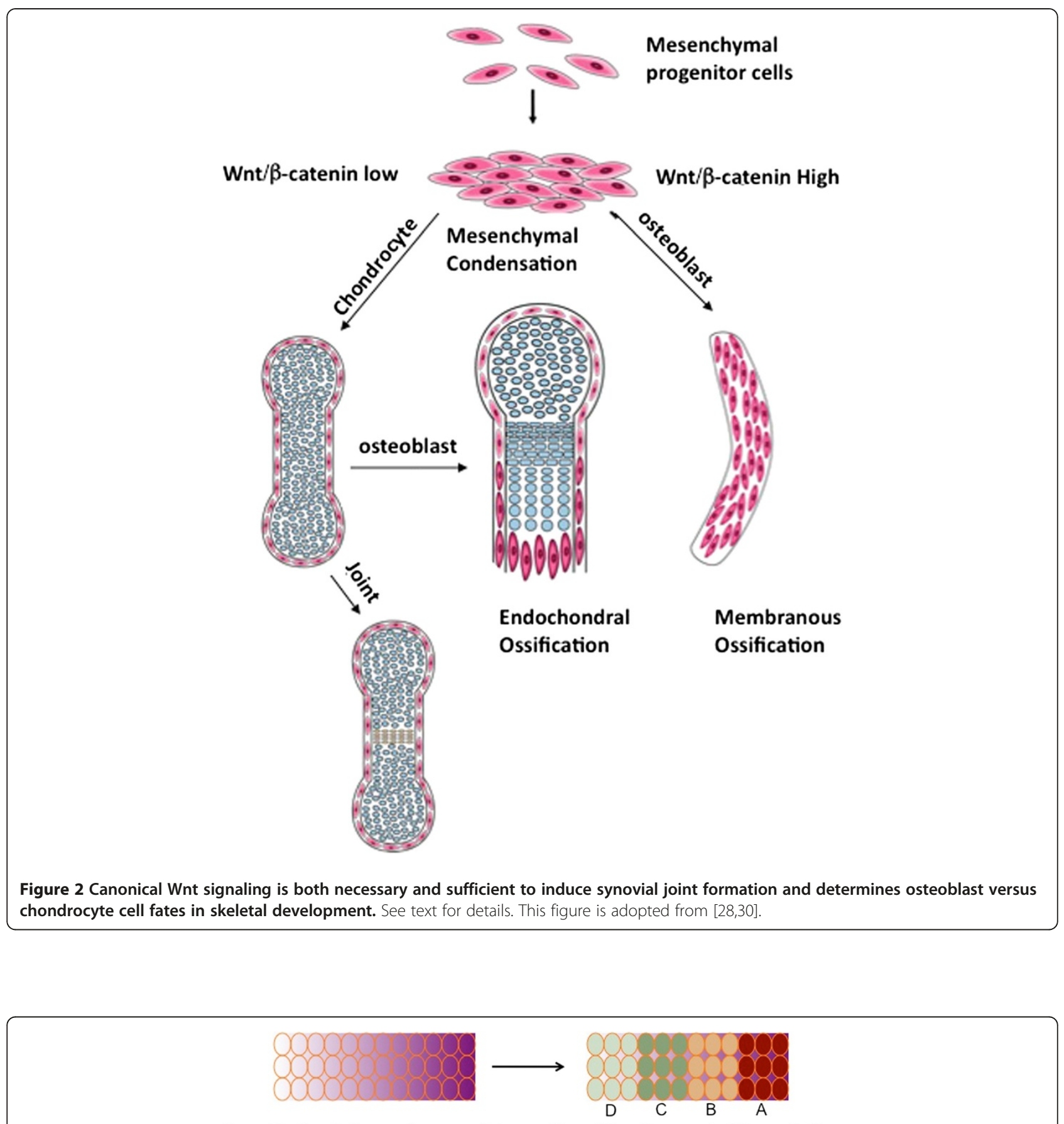

Quantitative information regulates cell proliferation and differentiation

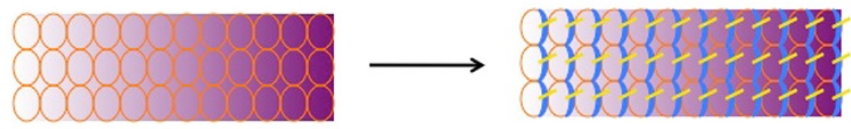

Directional information establishes cell polarity

Figure 3 Two fundamental roles of morphogen gradients in development. It is well known that morphogen gradients provide quantitative information to coordinate proliferation with differentiation. For instance, morphogen gradients induce the production of different cell fates (A, B, C, D) according to thresh hold concentrations of the morphogen. Morphogen gradients may also integrate directional information by controlling planar cell polarity indicated by uniform asymmetrical localization of key polarity protein (shown in blue) and cellular behavior like the uniform orientation of cell extensions like cilia (yellow). 
well known that a field of hundred of cells can acquire uniform planar cell polarity (PCP) which originally refers to the polarity in a plane perpendicular to the apical-basal axis [13,42-45]. PCP is evidenced by uniform asymmetrical localization of key polarity protein and uniform asymmetrical cellular behaviors such as the localization and orientation of cell extensions like cilia. Examples of PCP regulated processes are uniform orientations of Drosophila hair, mouse fur, inner ear sensory hair cells, elongating anteroposterior body axis and closing neural tubes. In a context dependent manner, the directional information provided by PCP is essential for cells to decide in which direction to divide, to move, or to interact with each other. As a class of critical morphogens, we thought Wnts may not jot just control cell proliferation and cell differentiation, they may also integrate the directional information by regulating $\mathrm{PCP}$. But the underlying mechanism remained elusive. For many years it was not shown that morphogens can act as global cues to convey directional information via PCP $[46,47]$. Work from our laboratory was the first two show that secreted Wnts can establish directional information via the PCP pathway.

The PCP pathway is best characterized in Drosophila in which a group of core PCP components were identified and they play conserved roles in both invertebrates and vertebrates $[13,42,43]$. These core PCP components include Wnt receptor frizzled, a four transmembrane protein Van Gogh (vang) (the vertebrate homologues are Van Gogh like 1 and 2 (Vangl 1 and 2)), flamingo (the vertebrate homologues are Celsr1, 2 and 3), prickle and disheveled (Figure 1). Wnt ligands have not been found to regulate PCP in Drosophila. However, in vertebrates such the zebrafish, Wnt11 and Wnt5a are both required to regulate PCP during convergent extension $[48,49]$. Downstream of the PCP core components, the PCP pathway might act through RhoA to control cytoskeleton remodeling and Jun kinase pathway to regulate gene expression, which still awaits rigorous genetic tests.

To investigate the function and mechanism of PCP, we removed both Vangl1 and Vangl2 as they form the smallest family of core PCP components, so the problem of functional redundancy within a gene family can be managed relatively easily. In addition, unlike Frizzled and Disheveled, Vangl1 and 2 are not shared by the canonical Wnt pathway. Therefore, the phenotypic perturbation in Vangl1 and 2 mutants should be solely due to altered PCP. In mammals, PCP has been found to play fundamental roles in development. For instance, loss of PCP in the mouse Vangl2 Looptail $(L p)$ mutant leads to randomization of the inner ear hair cells and open neural tube [45,50-55]. Mild condition of incomplete neural tube closure called spina bifida. In humans, spina bifida is the most common neural tube defect and can be caused by mutations in both human VANGL1 and 2
$[56,57]$. Since the nature of the Vangl2 Lp mutation was not clear and the $L p$ homozygous mutant is embryonic lethal at the time, we generated null alleles of Vangl1 and Vangl2 and a floxed allele of Vangl2 [51]. Both Vangl1 and 2 regulates embryonic development as Vangl1/2 double mutants are more severe than Vangl2 the single mutant and Vangl1 single mutant has no embryonic defects [51]. The various abnormalities demonstrated by the Vangl1/2 double mutants indicate that PCP is fundamentally important in many developmental processes.

The earliest defect we can find in the Vangl1/2 double mutant is in the establishment of left-right (L-R) asymmetry [51]. One of the molecular markers that allows one to detect left-right asymmetry in early embryos before any distinct morphological sign of left-right asymmetry shows up is unilateral expression of Nodal in the left lateral plate mesoderm of the early E8.5 mouse embryo [58,59]. In the Vangl1/2 double mutant, Nodal expression is randomized indicating that L-R asymmetry is randomized and Vangl1/2 mediated PCP acts upstream of Nodal expression to control L-R asymmetry.

The unilateral Nodal expression has to be triggered by an earlier event that breaks the bilateral symmetry. During vertebrate morphogenesis, establishment of L-R asymmetry follows the determination of dorsal-ventral (D-V) and anterior-posterior (A-P) body axes during gastrulation. In mice, bilateral symmetry is broken by a leftward fluid flow across a pit-like, teardrop-shaped node generated by posteriorly localized motile cilia of the node cells [60-63]. Both genetic analysis and mathematical modeling have shown that such leftward nodal flow is both necessary and sufficient to trigger unilateral Nodal expression. Abnormal cilium localization will result in turbulent nodal flow, which disrupts left-right patterning. Therefore, failure to transmit the A-P positional information in the node cells is likely to cause random cilium positioning and randomized L- $\mathrm{R}$ patterning. Thus, a mechanism that enables the node cells to interpret A-P positional information to position the nodal cilia sits at the top of the regulatory hierarchy of L-R asymmetry.

We found that the node cells are polarized by PCP along the A-P axis [51] (Figure 4). Both Vangl1 and Vangl2 protein were found to be selectively upregulated and asymmetrically localized in the forming node cells along the A-P axis. Outside of the node or prior to node formation, Vangl1 and Vangl2 protein levels were much lower and they were not asymmetrically localized. To test the functional significance of Vangl1/2 in node cell polarization, ciliogenesis and cilium localization were examined. In contrast to some previously published results in other systems, stringent genetic tests clearly show that PCP is not required for ciliogenesis [51,54]. However, in the Vangl or other PCP mouse mutants, cilia 

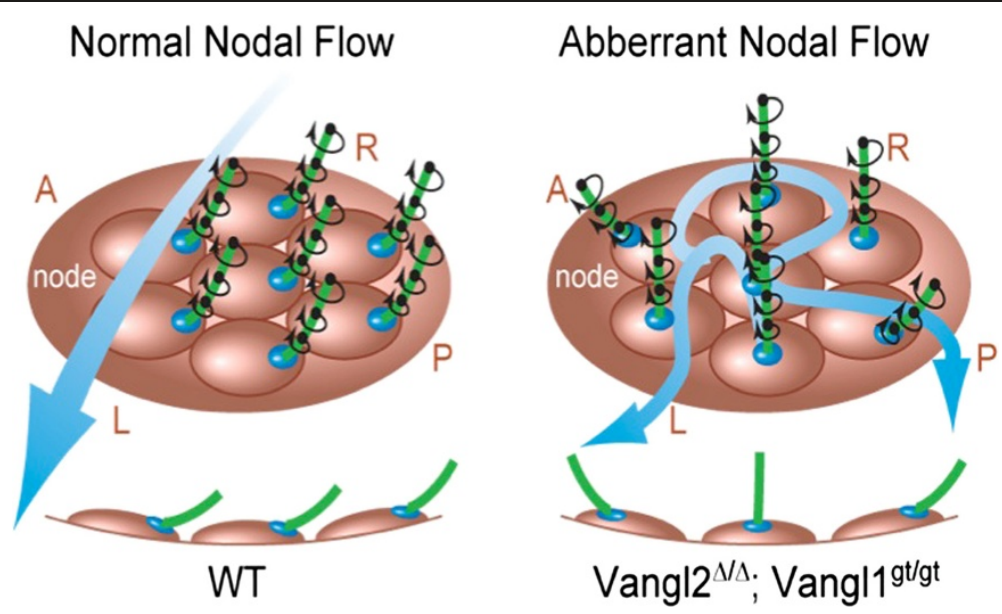

Figure 4 PCP breaks bilateral symmetry during left-right patterning by positioning the nodal cilia to the posterior side of the node cells. See text for details. This figure is adopted from (Song et al., 2010).

are randomly distributed around the center of the node cells, although Nodal cilia are mostly posteriorly localized in wild type embryos [51,54] (Figure 4). When the nodal flow is examined by following the paths of beads applied to the live embryos through live imaging, it was further discovered that randomization of nodal cilium localization leads to turbulent nodal flow [51,53] (Figure 4). In the control node, the bead paths were smooth and parallel to each other indicating the nodal flow is unidirectional. In the mutant, the bead paths contained abrupt turns, knots and often crossed with each other demonstrating the nodal flow is turbulent. Therefore, PCP is required to break the bilateral symmetry by establishing a unidirectional Nodal flow (Figure 4).

We then asked what establishes PCP in the developing embryo and whether PCP also regulates other important directional morphogenetic events such as limb elongation. Because the limb of the Vangl double mutant embryo is much shorter and distal digits were lost, we hypothesized that PCP controls directional limb elongation. Because $W n t 5 a$ is expressed in a graded manner in the early developing limb and primitive streak adjacent to the node and $W n t 5 a^{-/-}$mutant embryos exhibits much shortened limb [25,64], we also hypothesized Wnt5a is a cue that establishes PCP in the embryo. Indeed, Wnt5a genetically interacts with Vangl2 in neural tube closure and hair cell orientation in inner ear [16].

We then asked whether Wnt5a directs P-D limb elongation of the developing limb by regulating PCP. In the wild type limb, cartilage formation extends distally. But in the $W n t 5 a^{-/-}$distal limb, cartilage failed to extend distally, forming ball-like structure $[17,64]$. We found previously that loss of distal digits in the Wnt5 $a^{-/-}$embryo may be caused by upregulation of the anticondrogenic canonical Wnt signaling activity [25]. The ball-like structure of the forming digit cartilage led us to hypothesize that Wnt5a may also control cartilage elongation along the PD axis in limb development by regulating PCP.

To test whether the early differentiated chondrocytes are indeed polarized and show PCP, we examined Vangl2 protein localization and found that Vangl2 was asymmetrically localized along the P-D axis only in the Sox9-positive chondrocytes, not in the Sox9-negative interdigital mesenchymal cells in the limb [17]. These results demonstrate for the first time with a definitive molecular marker that chondrocytes are indeed polarized by PCP. Importantly, in the $W n t 5 a^{-1-}$ mutant limb, such polarized Vangl2 localization disappeared indicating that Wnt5a controls chondocyte polarity through PCP [17].

As Wnt5a genetically interacts with Vangl2 and Wnt5a has been found to bind Ror2, a receptor tyrosine kinase $[16,65]$, we further hypothesized that Wnt5a transduces its signal through a novel receptor complex containing Vangl2 and Ror2. Mutations in both WNT5A and ROR2 are found to cause Robinow syndrome characterized by shortened limb dwarfism [8-11]. To test this hypothesis further, we generated Ror2 and Vangl2 double mutant embryos and found that they phenocopied the Wnt5 $a^{-1-}$ embryo in the limb, craniofacial processes and the tail. The limb phenotypes of the Wnt5a mutants and the Ror2/Vangl2 double mutants are almost identical [17]. In addition, we found in the developing limb bud, Vangl2 antibodies can pull down Ror2. Furthermore, Vangl2 and Ror2 association is significantly enhanced by Wnt5a [17].

Then we asked how Wnt5a gradient leads to establishment of PCP. In another word, how the cells in the limbs sense the Wnt5a morphogen gradient and interpret the directional information [17]. We found that Wnt5a and 
many other Wnt ligands, when coexpressed with Ror2, enhanced Vangl2 phosphorylation. Expression of Wnt5a or Ror 2 alone also enhanced Vangl2 phorsphorylation to a lesser extent. The smear gel mobility shift pattern suggests that there are hyperphosphorylated and hypophosphrylated Vangl2, indicating that Vangl2 can be phosphorylated on multiple sites. Vangl2 phosphorylation is also detected in vivo in mouse tissues including the limb and brain [17]. Phosphorylation is abolished by calf intestinal phosphatase (CIP) treatment. In addition, Wnt5a -induced Vangl2 phosphorylation is much reduced in $R o r 2^{-/-}$MEF cells, indicating that Wnt5a induces Vangl2 phosphorylation through Ror2 [17].

To further understand how Vangl2 phosphorylation is regulated by Wnt5a/Ror2 signaling, we took a great effort to map the phosphorylation sites of Vangl2 [17]. We found that phosphorylation of Vangl2 occurs on conserved Serine (Ser) and threonine (Thr) residues that are organized in two different clusters in the protein. We also demonstrated that phosphorylation within both clusters occurs in a progressive manner such that founder residues are phosphorylated before others [17]. The progressive nature of Vangl2 phosphoylation suggests that different levels of Vangl2 phosphorylation is regulated by distinct Wnt5a dosages and this may be an underlying mechanism whereby responding cells interpret Wnt5a dosages. Because in the developing limb bud, there is a Wnt5a expression gradient from distal to proximal sides, we tested this in vivo first in the limb bud. We dissected the limb bud into distal parts with higher Wnt5a expression, the middle parts with medium Wnt5a expression and proximal parts with lower Wnt5a expression. Indeed, we found that there is a progressive reduction of Vangl2 phosphoryation distal to proximal limb bud [17]. Importantly, in the $R o r 2^{-/}$and $W n t 5 a^{-/-}$distal limb bud, hyperphosphorylated forms of Vangl2 were replaced by hypophosphorylated forms that are observed in proximal parts of limb bud. To further test that different levels of Vangl2 phosphorylation are induced by distinct dosages of Wnt5a, cells expressing Vangl2 and Ror2 were cocultured with various numbers of Wnt5aexpressing cells. Indeed, increasing Wnt5a dosages led to progressively more extensive Vangl2 phosphorylation [17]. These in vivo and in vitro studies demonstrate that regulating levels of Vangl2 phosphorylation is a way to sense the Wnt5a morphogen gradient.

We then tested whether phosphorylation regulates Vangl2 activity. We took advantage of the zebrafish trilobite mutants that are caused by Vangl 2 null mutations and exhibit convergent extension (CE) defects [49]. Wild type mouse Vangl 2 can rescue CE defects in $57 \%$ of the injected mutant fish embryos, a mutant vangl 2 with S84A mutation (a founder site) rescued $31 \%$ of inject mutant embryos, whereas all phosphomutant had no rescuing ability. A Vangl2 phospho-mimicking mutant showed increased rescuing ability. Therefore, phosphorylation is required for Vangl2 activity and higher levels of Vangl2 phosphorylation led to higher Vangl2 activities. In the developing limb bud, by inducing different levels of Vangl2 phosphorylation, Wnt5a gradient is translated into an activity gradient of Vangl2 (Figure 5). The limb chondrocytes can orient themselves by sensing an initial small difference in Vangl2 activities in its immediate neighbors. This difference is then amplified by cell-cell interactions and positive feedback loops such that Vangl2 protein aggregates only on one side of the plasma membrane, laying the ground for further asymmetric cellular behavior, such as directional cartilage formation and growth.

Apart from its critical role in controlling PCP, our lab also showed that Wnt5a can promote chondrocyte differentiation by inhibiting the canonical Wnt signaling activity [25]. Therefore, Wnt5a controls both cell proliferation and polarity by coordinating canonical Wnt signaling with Wnt/PCP signaling. It is possible that these two Wnt pathways are mutually inhibitory to each other [66]. In the case of skeletal development, it will be interesting to test whether altered bone morphology due to disrupted PCP
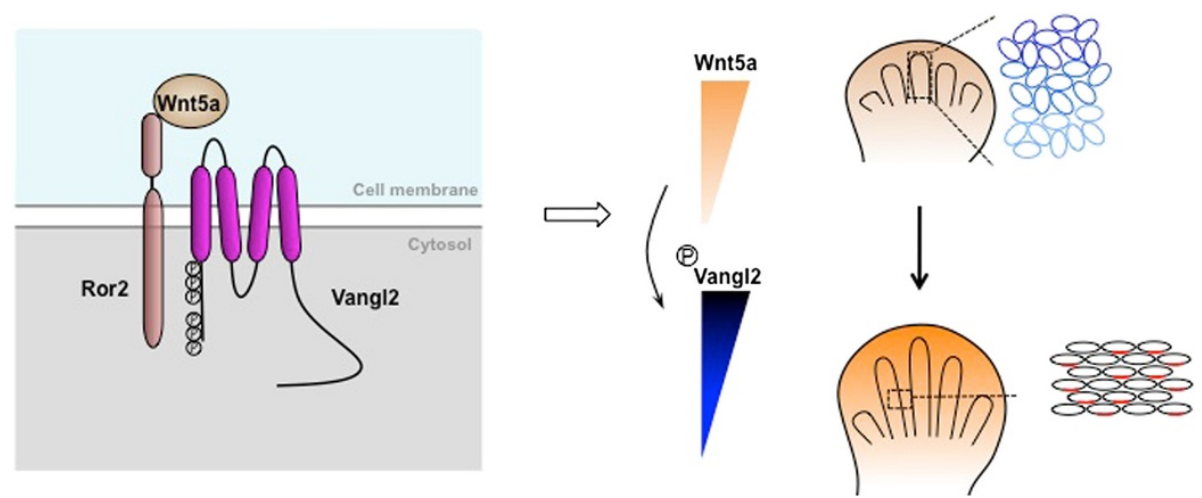

Figure 5 Model of Wnt5a signaling gradient in establishing planar cell polarity. See text for details. 
signaling also led to abnormal bone mass caused by alteration of canonical Wnt signaling.

\section{Acknowledgement}

This review is based on Dr Yingzi Yang's Young Investigator Award Lecture at the SCBA biennial meeting in Guangzhou, China, in 2011. Dr Yang would like to thank all current and previous members of the Yang lab for the hard work that led to this award. This work was supported by the Division of Intramural Research of the National Institutes of Health.

\section{Competing interests}

The author declares that she has no competing interests.

Received: 10 February 2012 Accepted: 20 April 2012

Published: 20 April 2012

\section{References}

1. Clevers H: Wnt/beta-catenin signaling in development and disease. Cell 2006, 127(3):469-480.

2. van Amerongen $R$, Nusse $R$ : Towards an integrated view of Wnt signaling in development. Development 2009, 136(19):3205-3214.

3. Angers S, Moon RT: Proximal events in Wnt signal transduction. Nat Rev Mol Cell Biol 2009, 10(7):468-477.

4. Nusse R, Varmus HE: Many tumors induced by the mouse mammary tumor virus contain a provirus integrated in the same region of the host genome. Cell 1982, 31(1):99-109.

5. Gong Y, Slee RB, Fukai N, Rawadi G, Roman-Roman S, Reginato AM, Wang H, Cundy T, Glorieux FH, Lev D, et al: LDL receptor-related protein 5 (LRP5) affects bone accrual and eye development. Cell 2001, 107(4):513-523.

6. Little RD, Carulli JP, Del Mastro RG, Dupuis J, Osborne M, Folz C, Manning SP, Swain PM, Zhao SC, Eustace B, et al: A mutation in the LDL receptor-related protein 5 gene results in the autosomal dominant high-bone-mass trait. Am J Hum Genet 2002, 70(1):11-19.

7. Boyden LM, Mao J, Belsky J, Mitzner L, Farhi A, Mitnick MA, Wu D, Insogna K, Lifton RP: High bone density due to a mutation in LDL-receptor-related protein 5. N Engl J Med 2002, 346(20):1513-1521.

8. van Bokhoven H, Celli J, Kayserili H, van Beusekom E, Balci S, Brussel W, Skovby F, Kerr B, Percin EF, Akarsu N, et al: Mutation of the gene encoding the ROR2 tyrosine kinase causes autosomal recessive Robinow syndrome. Nat Genet 2000, 25(4):423-426.

9. Afzal AR, Rajab A, Fenske CD, Oldridge M, Elanko N, Ternes-Pereira E, Tuysuz $B$, Murday VA, Patton MA, Wilkie AO, et al: Recessive Robinow syndrome, allelic to dominant brachydactyly type B, is caused by mutation of ROR2. Nat Genet 2000, 25(4):419-422.

10. Schwabe GC, Tinschert S, Buschow C, Meinecke P, Wolff G, Gillessen-Kaesbach G, Oldridge M, Wilkie AO, Komec R, Mundlos S: Distinct mutations in the receptor tyrosine kinase gene ROR2 cause brachydactyly type B. Am J Hum Genet 2000, 67(4):822-831.

11. Person AD, Beiraghi $S$, Sieben $C M$, Hermanson $S$, Neumann AN, Robu ME, Schleiffarth JR, Billington CJ Jr, Van Bokhoven H, Hoogeboom JM, et al: WNT5A mutations in patients with autosomal dominant Robinow syndrome. Dev Dyn 2010, 239(1):327-337.

12. Slusarski DC, Yang-Snyder J, Busa WB, Moon RT: Modulation of embryonic intracellular Ca2+ signaling by Wnt-5A. Dev Biol 1997, 182(1):114-120.

13. Tree $D R, M a D, A x e l r o d ~ J D: A$ three-tiered mechanism for regulation of planar cell polarity. Semin Cell Dev Biol 2002, 13(3):217-224.

14. Gros J, Serralbo O, Marcelle C: WNT11 acts as a directional cue to organize the elongation of early muscle fibres. Nature 2009, 457(7229):589-593.

15. Heisenberg CP, Tada M, Rauch GJ, Saude L, Concha ML, Geisler R, Stemple DL, Smith JC, Wilson SW: Silberblick/Wnt11 mediates convergent extension movements during zebrafish gastrulation. Nature 2000, 405(6782):76-81.

16. Qian D, Jones C, Rzadzinska A, Mark S, Zhang X, Steel KP, Dai X, Chen P: Wnt5a functions in planar cell polarity regulation in mice. Dev Biol 2007, 306(1):121-133.

17. Gao B, Song H, Bishop K, Elliot G, Garrett L, English MA, Andre P, Robinson J, Sood R, Minami Y, et al: Wnt signaling gradients establish planar cell polarity by inducing Vangl2 phosphorylation through Ror2. Developmental cell 2011, 20(2):163-176.

18. Gros J, Hu JK, Vinegoni C, Feruglio PF, Weissleder R, Tabin CJ: WNT5A/JNK and FGF/MAPK pathways regulate the cellular events shaping the vertebrate limb bud. Curr Biol 2010, 20(22):1993-2002.

19. Wang B, Sinha T, Jiao K, Serra R, Wang J: Disruption of PCP signaling causes limb morphogenesis and skeletal defects and may underlie Robinow syndrome and brachydactyly type B. Hum Mol Genet 2011, 20(2):271-285.

20. Wyngaarden LA, Vogeli KM, Ciruna BG, Wells M, Hadjantonakis AK, Hopyan $\mathrm{S}$ : Oriented cell motility and division underlie early limb bud morphogenesis. Development 2010, 137(15):2551-2558.

21. Zelzer E, Olsen BR: The genetic basis for skeletal diseases. Nature 2003, 423(6937):343-348.

22. Yang Y: Skeletal morphogenesis during embryonic development. Crit Rev Eukaryot Gene Expr 2009, 19(3):197-218.

23. Karsenty G, Oury F: Biology Without Walls: The Novel Endocrinology of Bone. Annu Rev Physiol 2011, 74:87-105.

24. Yang $Y$, Topol L, Lee $H$, Wu J: Wnt5a and Wnt5b exhibit distinct activities in coordinating chondrocyte proliferation and differentiation. Development 2003, 130(5):1003-1015.

25. Topol L, Jiang X, Choi H, Garrett-Beal L, Carolan PJ, Yang Y: Wnt-5a inhibits the canonical Wnt pathway by promoting GSK-3-independent \{beta\}-catenin degradation. J Cell Biol 2003, 162(5):899-908.

26. Topol L, Chen W, Song H, Day TF, Yang Y: Sox9 inhibits Wnt signaling by promoting beta-catenin phosphorylation in the nucleus. I Biol Chem 2009, 284(5):3323-3333.

27. Mak KK, Chen MH, Day TF, Chuang PT, Yang Y: Wnt/beta-catenin signaling interacts differentially with Ihh signaling in controlling endochondral bone and synovial joint formation. Development 2006, 133(18):3695-3707.

28. Guo X, Day TF, Jiang X, Garrett-Beal L, Topol L, Yang Y: Wnt/beta-catenin signaling is sufficient and necessary for synovial joint formation. Genes Dev 2004, 18(19):2404-2417.

29. Guo X, Mak KK, Taketo MM, Yang Y: The Wnt/beta-catenin pathway interacts differentially with PTHrP signaling to control chondrocyte hypertrophy and final maturation. PLoS One 2009, 4(6):e6067.

30. Day TF, Guo X, Garrett-Beal L, Yang Y: Wnt/beta-catenin signaling in mesenchymal progenitors controls osteoblast and chondrocyte differentiation during vertebrate skeletogenesis. Dev Cell 2005, 8(5):739-750

31. Storm EE, Huynh TV, Copeland NG, Jenkins NA, Kingsley DM, Lee SJ: Limb alterations in brachypodism mice due to mutations in a new member of the TGF beta-superfamily. Nature 1994, 368(6472):639-643.

32. Loughlin J, Dowling B, Chapman K, Marcelline L, Mustafa Z, Southam L, Ferreira A, Ciesielski C, Carson DA, Corr M: Functional variants within the secreted frizzled-related protein 3 gene are associated with hip osteoarthritis in females. Proc Natl Acad Sci U S A 2004, 101(26):9757-9762.

33. Agholme F, Li X, Isaksson H, Ke HZ, Aspenberg P: Sclerostin antibody treatment enhances metaphyseal bone healing in rats. Journal of bone and mineral research: the official journal of the American Society for Bone and Mineral Research 2010, 25(11):2412-2418.

34. Li X, Ominsky MS, Warmington KS, Morony S, Gong J, Cao J, Gao Y, Shalhoub V, Tipton B, Haldankar R, et al: Sclerostin antibody treatment increases bone formation, bone mass, and bone strength in a rat model of postmenopausal osteoporosis. Journal of bone and mineral research: the official journal of the American Society for Bone and Mineral Research 2009, 24(4):578-588

35. Li X, Ominsky MS, Warmington KS, Niu QT, Asuncion FJ, Barrero M, Dwyer D, Grisanti M, Stolina M, Kostenuik PJ, et al: Increased bone formation and bone mass induced by sclerostin antibody is not affected by pretreatment or cotreatment with alendronate in osteopenic, ovariectomized rats. Endocrinology 2011, 152(9):3312-3322.

36. Li X, Warmington KS, Niu QT, Asuncion FJ, Barrero M, Grisanti M, Dwyer D, Stouch B, Thway TM, Stolina M, et al: Inhibition of sclerostin by monoclonal antibody increases bone formation, bone mass, and bone strength in aged male rats. Journal of bone and mineral research: the official journal of the American Society for Bone and Mineral Research 2010, 25(12):2647-2656.

37. Lawrence PA: Morphogens: how big is the big picture? Nat Cell Biol 2001, 3(7):E151-154

38. Turing AM: The chemical basis of morphogenesis. Philosophical Transactions of the Royal Society of London B 1952, 237(641):37-72. 
39. Wolpert L: Positional information and the spatial pattern of cellular differentiation. J Theor Biol 1969, 25(1):1-47.

40. Benazet JD, Zeller R: Vertebrate limb development: moving from classical morphogen gradients to an integrated 4-dimensional patterning system. Cold Spring Harbor perspectives in biology 2009, 1(4):a001339.

41. Towers $M$, Tickle C: Growing models of vertebrate limb development. Development 2009, 136(2):179-190.

42. Seifert JR, Mlodzik M: Frizzled/PCP signalling: a conserved mechanism regulating cell polarity and directed motility. Nat Rev Genet 2007, 8(2):126-138.

43. Wang $Y$, Nathans J: Tissue/planar cell polarity in vertebrates: new insights and new questions. Development 2007, 134(4):647-658.

44. Zallen JA: Planar polarity and tissue morphogenesis. Cell 2007, 129(6):1051-1063.

45. McNeill H: Planar cell polarity: keeping hairs straight is not so simple. Cold Spring Harb Perspect Biol 2010, 2(2):a003376.

46. Lawrence PA, Struhl G, Casal J: Planar cell polarity: one or two pathways? Nat Rev Genet 2007, 8(7):555-563.

47. Strutt D: Gradients and the specification of planar polarity in the insect cuticle. Cold Spring Harb Perspect Biol 2009, 1(5):a000489.

48. Rauch GJ, Hammerschmidt M, Blader P, Schauerte HE, Strahle U, Ingham PW, McMahon AP, Haffter P: Wnt5 is required for tail formation in the zebrafish embryo. Cold Spring Harb Symp Quant Biol 1997, 62:227-234.

49. Jessen JR, Topczewski J, Bingham S, Sepich DS, Marlow F, Chandrasekhar A Solnica-Krezel L: Zebrafish trilobite identifies new roles for Strabismus in gastrulation and neuronal movements. Nat Cell Biol 2002, 4(8):610-615.

50. Axelrod JD: Progress and challenges in understanding planar cell polarity signaling. Semin Cell Dev Biol 2009, 20(8):964-971.

51. Song H, Hu J, Chen W, Elliott G, Andre P, Gao B, Yang Y: Planar cell polarity breaks bilateral symmetry by controlling ciliary positioning. Nature 2010, 466(7304):378-382.

52. Guirao B, Meunier A, Mortaud S, Aguilar A, Corsi JM, Strehl L, Hirota Y, Desoeuvre A, Boutin C, Han YG, et al: Coupling between hydrodynamic forces and planar cell polarity orients mammalian motile cilia. Nat Cell Biol 2010, 12(4):341-350.

53. Hashimoto M, Shinohara K, Wang J, Ikeuchi S, Yoshiba S, Meno C, Nonaka S, Takada S, Hatta K, Wynshaw-Boris A, et al: Planar polarization of node cells determines the rotational axis of node cilia. Nat Cell Biol 2010, 12(2):170-176

54. Borovina A, Superina S, Voskas D, Ciruna B: Vangl2 directs the posterior tilting and asymmetric localization of motile primary cilia. Nat Cell Biol 2010, 12(4):407-412

55. Torban E, Patenaude AM, Leclerc S, Rakowiecki S, Gauthier S, Andelfinger G, Epstein DJ, Gros P: Genetic interaction between members of the Vangl family causes neural tube defects in mice. Proc Natl Acad Sci U S A 2008, 105(9):3449-3454.

56. Kibar Z, Torban E, McDearmid JR, Reynolds A, Berghout J, Mathieu M, Kirillova I, De Marco P, Merello E, Hayes JM, et al: Mutations in VANGL1 associated with neural-tube defects. N Engl J Med 2007, 356(14):1432-1437.

57. Lei YP, Zhang $T$, Li H, Wu BL, Jin L, Wang HY: VANGL2 mutations in human cranial neural-tube defects. N Engl J Med 2010, 362(23):2232-2235.

58. Lowe LA, Supp DM, Sampath K, Yokoyama T, Wright CV, Potter SS, Overbeek $P$, Kuehn MR: Conserved left-right asymmetry of nodal expression and alterations in murine situs inversus. Nature 1996, 381(6578):158-161.

59. Collignon J, Varlet I, Robertson EJ: Relationship between asymmetric nodal expression and the direction of embryonic turning. Nature 1996, 381(6578):155-158

60. Blum M, Andre P, Muders K, Schweickert A, Fischer A, Bitzer E, Bogusch S, Beyer T, van Straaten HW, Viebahn C: Ciliation and gene expression distinguish between node and posterior notochord in the mammalian embryo. Differentiation 2007, 75(2):133-146.

61. Nonaka S, Yoshiba S, Watanabe D, Ikeuchi S, Goto T, Marshall WF, Hamada $\mathrm{H}$ : De novo formation of left-right asymmetry by posterior tilt of nodal cilia. PLOS Biol 2005, 3(8):e268.

62. Okada Y, Takeda S, Tanaka Y, Belmonte JC, Hirokawa N: Mechanism of nodal flow: a conserved symmetry breaking event in left-right axis determination. Cell 2005, 121(4):633-644.

63. Nonaka S, Tanaka Y, Okada Y, Takeda S, Harada A, Kanai Y, Kido M, Hirokawa $\mathrm{N}$ : Randomization of left-right asymmetry due to loss of nodal cilia generating leftward flow of extraembryonic fluid in mice lacking KIF3B motor protein. Cell 1998, 95(6):829-837.

64. Yamaguchi TP, Bradley A, McMahon AP, Jones S: A Wnt5a pathway underlies outgrowth of multiple structures in the vertebrate embryo. Development 1999, 126(6):1211-1223.

65. Oishi I, Suzuki H, Onishi N, Takada R, Kani S, Ohkawara B, Koshida I, Suzuki K, Yamada G, Schwabe GC, et al: The receptor tyrosine kinase Ror2 is involved in non-canonical Wnt5a/JNK signalling pathway. Genes Cells 2003, 8(7):645-654

66. Bryja V, Andersson ER, Schambony A, Esner M, Bryjova L, Biris KK, Hall AC, Kraft B, Cajanek L, Yamaguchi TP, et al: The extracellular domain of Lrp5/6 inhibits noncanonical Wnt signaling in vivo. Molecular biology of the cell 2009, 20(3):924-936

doi:10.1186/2045-3701-2-14

Cite this article as: Yang: Wnt signaling in development and disease. Cell \& Bioscience 2012 2:14.

\section{Submit your next manuscript to BioMed Central and take full advantage of:}

- Convenient online submission

- Thorough peer review

- No space constraints or color figure charges

- Immediate publication on acceptance

- Inclusion in PubMed, CAS, Scopus and Google Scholar

- Research which is freely available for redistribution 